\title{
Managing Life-Threatening Malignant Superior Mediastinal Syndrome in Pregnancy: When Benefits of Radiation and Chemotherapy Outweigh the Risks-A Case Report and Review of Literature
}

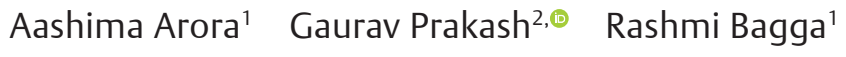 \\ 1Department of Obstetrics and Gynaecology, Post Graduate \\ Institute of Medical Education and Research, Chandigarh, \\ Chandigarh, India \\ 2Department of Internal Medicine, Post Graduate Institute of \\ Medical Education and Research, Chandigarh, Chandigarh, India \\ ${ }^{3}$ Department of Cytopathology, Post Graduate Institute of Medical \\ Education and Research, Chandigarh, Chandigarh, India
}

\author{
Radhika Srinivasan ${ }^{3} \quad$ Arihant Jain ${ }^{2, \odot}$
}

Address for correspondence Arihant Jain, MD,DM, Department of Internal Medicine, PGIMER, Chandigarh 160012, India (e-mail: drarihantjain86@gmail.com).

Ind J Med Paediatr Oncol:2021;42:208-212.

\begin{abstract}
There is scarce literature on managing superior mediastinal syndrome during pregnancy. We report a case of 26-year-old primigravida who presented with life-threatening superior mediastinal syndrome at 32 weeks of gestation. The diagnosis was significantly delayed and, as a result, she reached the emergency with stridor and impending respiratory failure. She was diagnosed with primary mediastinal B cell lymphoma Lugano Stage II with a bulky mediastinal mass. She was treated with chemoimmunotherapy and underwent a preterm vaginal delivery after a week. She delivered a $1.6 \mathrm{~kg}$ healthy child with no malformations. Later, she completed three cycles of rituximab, cyclophosphamide, vincristine, doxorubicin, and prednisolone and five cycles of dose-adjusted etoposide, prednisolone, vincristine, cyclophosphamide, doxorubicin,

Keywords

- pregnancy

- lymphoma

- radiation

- chemotherapy rituximab, followed by radiotherapy. She continues to be in remission at 18 months of follow-up. Delaying diagnostic imaging that involves ionizing radiation exposure and chemotherapy to avoid teratogenic and obstetric complications during pregnancy can adversely affect the prognosis in certain patients with high-grade malignancies. On the contrary, prompt multidisciplinary management can lead to a gratifying outcome.
\end{abstract}

\section{Introduction}

With the average age of pregnancy increasing globally, the prevalence of cancer in pregnancy is also likely to increase. Currently, cancer is diagnosed in $0.1 \%$ of all pregnancies. ${ }^{1}$ Data regarding the clinical characteristics and

DOI https://doi.org/ $10.1055 / \mathrm{s}-0041-1732819$ ISSN 0971-5851 outcomes of superior mediastinal syndrome during pregnancy is limited. ${ }^{2,3}$ An accurate and timely diagnosis, along with appropriate staging of the malignancy, is essential for achieving an optimal outcome. The tendency to avoid radiation exposure for diagnostic imaging and the administration of chemotherapy for treatment of malignancy throughout (c) 2021. Indian Society of Medical and Paediatric Oncology.

This is an open access article published by Thieme under the terms of the Creative Commons Attribution-NonDerivative-NonCommercial-License, permitting copying and reproduction so long as the original work is given appropriate credit. Contents may not be used for commercial purposes, or adapted, remixed, transformed or built upon. (https://creativecommons.org/licenses/by-nc-nd/4.0/).

Thieme Medical and Scientific Publishers Private Ltd. A-12, Second Floor, Sector -2, NOIDA -201301, India 
the entire gestational period may be harmful in certain cases of high-grade neoplasms. This is because delays in diagnosis and administration of curative intent chemotherapy itself can considerably increase the maternal morbidity as well as risk the fetus. With the advent of newer modalities of imaging (with limited or no radiation exposure) and the accumulating evidence for safety of chemotherapeutic drugs in select situations, successful management of pregnancy with cancers is now possible. Available data, although scarce, suggest feasibility of combination chemoimmunotherapy with optimal fetomaternal outcomes for primary mediastinal B cell lymphoma (PMBCL) in pregnancy. ${ }^{4}$

We successfully managed a pregnant young woman in whom the intention to avoid radiation exposure for a chest X-ray delayed the diagnosis of a malignant chest mass for 2 months. As a result, the patient landed up with a life-threatening superior mediastinal syndrome due to compression by a rapidly enlarging mediastinal lymphoma.

\section{Case Report}

A 26-year-old primigravida presented to our institute at 32 weeks of gestation with complaints of progressive dyspnea for one-and-a-half months, followed by orthopnea and chest pain for 2 weeks. There was a history of increasing weakness and loss of appetite, which were attributed to the pregnancy. She was initially advised bronchodilators, antihistamines, and antibiotics. Later, her echocardiography revealed moderate pericardial effusion. At this point, a chest X-ray was avoided due to fear of radiation exposure to the fetus. She was empirically started on antitubercular therapy with no improvement. Hence, she was referred to our institute. At presentation, her pulse rate was 140/min, Blood pressure was $100 / 60 \mathrm{~mm} \mathrm{Hg}$ with a pulse-paradox of $20 \mathrm{~mm} \mathrm{Hg}$. She had a respiratory rate of 40 per minute and room air saturation of $86 \%$ with edematous suffused face and nonpulsatile elevated jugular venous pulse. She had multiple dilated veins over her arms and anterior chest wall with flow from above downward. There was no palpable lymphadenopathy or hepatosplenomegaly and she had an Eastern Cooperative Oncology Group performance status of 4 . On auscultation, the heart sounds appeared muffled and there was decreased air entry in the anterior mammary areas. She was managed in a propped-up position, with oxygen and diuretics. Echocardiography ruled out massive pericardial effusion. She had a hemoglobin of $10.6 \mathrm{~g} / \mathrm{dL}$, total leucocyte count of 11,600 cells $/ \mu \mathrm{L}$, and platelet count of $2.6 \times 10^{6} / \mu \mathrm{L}$ with no circulating atypical cells. As the previous chest X-ray had revealed a mediastinal widening, a contrast-enhanced computed tomography (CT) scan of chest (with abdominal shielding) was done that revealed a heterogeneously enhancing soft tissue mass measuring $7.9 \times 10.1 \times 8.1 \mathrm{~cm}$ in the mediastinal/retrosternal region, encasing the ascending aorta, the arch of the aorta, the origins of major arteries, and the main pulmonary artery and its branches (-Fig. 1A, B). The mass was also encasing the trachea causing significant luminal narrowing. There was complete nonopacification of the superior vena cava and bilateral internal jugular veins
(IJV) with multiple tortuous collaterals seen along the chest wall. Obstetric ultrasonography (USG) revealed a single live fetus of $\sim 30$ weeks of gestation with reduced amniotic fluid and a biophysical profile score of $8 / 10$ with good fetal movements, breathing, tone, and heart rate pattern.

As the patient was unable to lie supine due to orthopnea, a CT-guided core biopsy was not feasible. Therefore, she underwent a bedside USG-guided fine-needle aspiration through a subcostal approach in the semirecumbent position. The smears showed many scattered large atypical lymphoid cells on a hemorrhagic background. The cells had scanty cytoplasm with fine vacuolation and large nuclei with opened-up chromatin with one or two peripheral nucleoli (-Fig. 2A). Upon flow cytometric immunophenotyping, these cells that were CD45+ were gated and showed strong positivity for B cell markers, CD19 and CD20 ( - Fig. 2B), along with human leukocyte antigen-DR. They were negative for CD5, CD10, CD23, other T cell markers including CD3, 4, 5, 8, CD34 and TdT. Light chain restriction was inconclusive. Cell block ( - Fig. 2C) for immunohistochemistry showed strong diffuse membranous positivity for $\mathrm{CD} 20$ ( - Fig. 2D) and weak positivity for $\mathrm{CD} 30$ ( - Fig. 2E) and CD10 (-Fig. 2F). The bone marrow examination was normal. Based upon her clinical and cytomorphological findings, a diagnosis of PMBCL, it was stage II bulky disease. Staging bone marrow is done as a part of protocol in all high grade Non-Hodgkin lymphoma (NHL), particularly if the PET is not done at baseline. After detailed discussion with the patient and the family, regarding the need for chemotherapy and its possible effects on the fetus, treatment was initiated with RCHOP (rituximab, cyclophosphamide, doxorubicin, vincristine, and prednisolone) along with a therapeutic dose of enoxaparin for anticoagulation. Fetal evaluation showed intrauterine growth restriction with oligohydramnios. She was followed up with biweekly biophysical profiles and nonstress testing. After 2 weeks of chemotherapy, her orthopnea had resolved. Ascertaining the resolution of tracheal compression was deemed necessary by the anesthetists as she might have required emergency cesarean section and intubation during labor. Repeat CT scan revealed partial reduction of the mediastinal mass to 7.5 $\times 10 \times 5.5 \mathrm{~cm}$ and resolution of the tracheal compression. At 3 weeks post-chemotherapy, following a course of
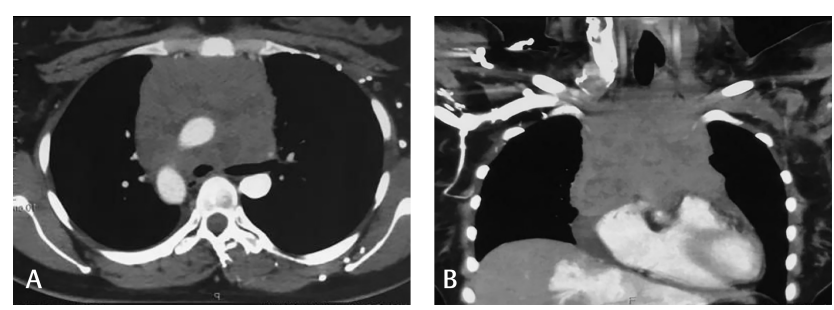

Fig. 1 (A) Axial image of contrast-enhanced computed tomography chest revealing a large lobulated heterogeneous solid mass in the anterior mediastinum, encasing the tracheobronchial tree and great vessels, with nonopacification of the superior vena cava; (B) Coronal image of contrast-enhanced computed tomography chest revealing a large lobulated heterogeneous solid mass in the anterior mediastinum, encasing the tracheobronchial tree and great vessels, with nonopacification of the superior vena cava. 

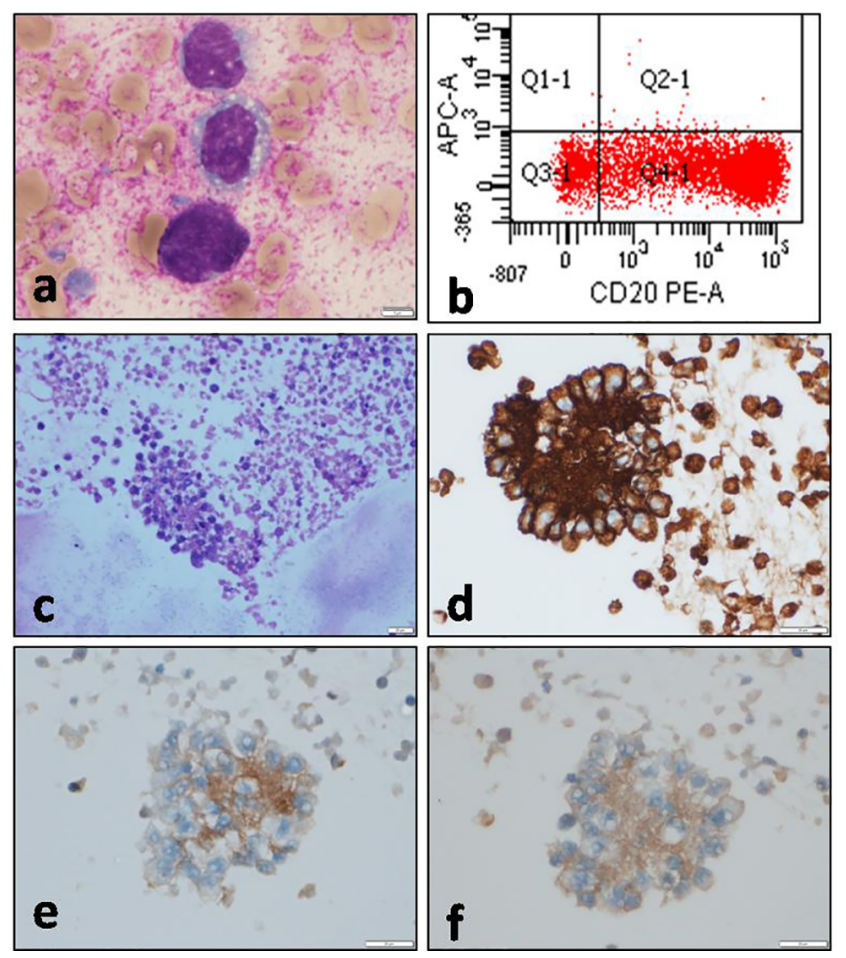

Fig. 2 Image panel of primary mediastinal B cell lymphoma. (A) Smear showing lymphoma cells. (B) Flow cytometric immunophenotyping showing CD20 positivity. (C) Cell block section (hematoxylin-eosin stain). (D-F) Cell block section showing CD20, CD30, and CD10 immunohistochemistry (immunoperoxidase stain).

antenatal corticosteroids for fetal lung maturity, labor was induced with a combined mechanical and pharmacological approach (intracervical Foley catheter plus intracervical prostaglandin E2 gel $0.5 \mathrm{mg}$ ) for cervical ripening for 12 hours, followed by oxytocin infusion. Pethidine was used for labor analgesia and she delivered vaginally $1.6 \mathrm{~kg}$ preterm, small for gestational age (weight less than 10th centile for gestational age) and at $35^{+1}$ weeks with Apgar score of 8 at 1 and 5 minutes. Breastfeeding was withheld and the baby did well on top feeds and did not require any neonatal intensive are. The patient initially received three cycles of standard dose RCHOP considering her peripartum status and preference for an outpatient regimen. The interim CT scan showed residual $5 \times 4 \times 5.5 \mathrm{~cm}$ mass. Therefore, the regimen was escalated to DA-EPOCH-R (dose adjusted etoposide, prednisolone, vincristine, cyclophosphamide, doxorubicin, rituximab). She received five additional cycles of DA-EPOCH-R. After discussing the added risk of cardiotoxicity and breast cancer versus a possible progression-free survival benefit, she was given $30 \mathrm{~Gy}$ (15 fractions over 3 weeks) involved-field radiotherapy, and attained complete remission. Both mother and child are doing well at a follow-up of 18 months from diagnosis.

\section{Discussion}

There is a significant paucity of awareness among the medical fraternity regarding the radiation risks to fetus when imaging pregnant women. ${ }^{5}$ Undoubtedly, modalities that do not use ionizing radiation, such as USG and magnetic resonance imaging (MRI), must be preferred for evaluating any condition in a pregnant lady as these have been consistently found to be safe. ${ }^{6}$ At the same time, it must be remembered that no imaging study should be withheld when deemed necessary for maternal safety. Frequently, fatigue, weight loss, low-grade fever, and abdominal discomfort are attributed to pregnancy itself rather than a malignancy. This frequently delays the diagnosis of cancers in pregnancy. ${ }^{7}$ Moreover, avoidance of X-rays or CT scans can further add to the diagnostic delay, as had happened in this case. We wish to reiterate that these inadvertent delays can lead to increased fetomaternal morbidity and mortality.

The effects of prenatal exposure to ionizing radiation can vary based on the dose and the length of gestation. There is significant data regarding the safety of single X-rays during pregnancy. ${ }^{8-10}$ The fetal effects of X-ray exposure may be in the form of teratogenesis (fetal malformations), carcinogenesis (induced malignancies), or mutagenesis (alteration of germline genes). With regard to teratogenesis, an embryo is most susceptible during organogenesis (2-7 weeks after conception) and in the early fetal period ( $8-15$ weeks after conception). Mental retardation and growth restriction, including microcephaly, are the most common malformations after significant radiation exposure. ${ }^{11}$ The teratogenic health effects are not observed until the radiation exposure crosses $0.05 \mathrm{~Gy}$ ( $5 \mathrm{rad}$ ) at any gestation; the threshold dose for teratogenicity increases to 10 to $20 \mathrm{rad}$ in at around 16 weeks of gestation, and up to 50 to 70 rad beyond 16 weeks. On the contrary, exposure to as little as 1 or 2 rad (which may be reached by some radiographic studies) has been associated with a slight increase in childhood malignancies, especially leukemia from a background risk of $\sim 3.6$ per 10,000 to 5 per $10,000 .{ }^{12}$ The actual fetal X-ray exposure in most diagnostic investigations is much lower than this threshold (8). Chest radiography (two views) exposes the fetus to less than $0.0001 \mathrm{~Gy}(<0.01 \mathrm{rad})$, while a contrast-enhanced CT abdomen (10 slices) typically has an estimated exposure of 0.00240 to $0.0260 \mathrm{~Gy}$ (0.240 to $2.60 \mathrm{rad}) .{ }^{12}$ Besides, there are concerns regarding the causation of germ-line mutations with fetal exposure to X-rays, potentially affecting future generations. ${ }^{13}$ Ionizing radiation increases the frequency of mutations occurring naturally in the general population. The radiation dose required to double the baseline mutation rate is between 50 and 100 rads. ${ }^{14}$ Most health care providers are highly sensitized to these risks associated with intra-uterine $\mathrm{X}$-ray exposure. At times this fear of radiation exposure to the fetus leads to an unrealistic avoidance of diagnostic imaging. However, the benefits accrued from the early diagnosis of a malignancy with the help of imaging in a pregnant female with suspected cancer outweigh the risk frequently. Therefore, using safe modalities such as USG and MRI and even X-rays and CT scan with adequate shielding maybe desirable in certain cases. The physicians must include the couple in the decision-making process whenever such situations arise. 
Lymphoma is the fourth most common malignancy diagnosed during pregnancy. ${ }^{7}$ Fine-needle aspiration played a pivotal role in establishing the diagnosis within a few hours in this case. The decision favoring the use of cytotoxic chemotherapy and targeted immunotherapy during pregnancy was based on the staging, the histologic subtype of tumor, and the gestational maturity of the fetus. In the first trimester, a wait and watch approach can be undertaken for low-grade lymphomas, whereas aggressive lymphomas often warrant a discussion with the family regarding the pros and cons of medical termination of pregnancy. However, in the secondand third-trimesters of pregnancy, there is significant literature to support the safety of RCHOP-based chemotherapy with favorable fetomaternal outcomes. ${ }^{15,16}$ In a retrospective analysis of 90 patients who were diagnosed with lymphoma during pregnancy, there were no differences in maternal complications, perinatal events, or median infant birth weight based on deferred versus antenatal therapy beyond the first trimester. ${ }^{17}$

In the current case, one cycle of RCHOP chemotherapy led to a marked improvement in the patient's orthopnea and the pregnancy could be safely prolonged for three more weeks, and fetal lung maturity was ensured. The current case supports the prior findings that in utero exposure to nonantimetabolite chemotherapy including anthracycline exposure in second and third trimesters is not known to affect fetomaternal outcomes including cardiac functions. ${ }^{18}$ However, we strongly believe that the significant delay in diagnosing the disease due to the avoidance of imaging after the onset of initial symptoms and the empiric use of anti-tubercular therapy hampered the timely institution of chemotherapy in the current case. This contributed to the progression of the lymphoma to a bulky stage II disease with a life-threatening superior mediastinal syndrome. As DA-EPOCH-R is associated with higher short-term treatment-related toxicities as well as requires frequent inpatient admissions; therefore, the patient initially received three cycles of RCHOP in the initial perinatal period. ${ }^{19}$ However, it was later escalated to DA-EPOCH-R considering the bulky nature of disease at baseline and suboptimal interim response. ${ }^{20,21}$ Currently, there is lack of robust evidence to omit radiotherapy in a patient with bulky high-grade lymphoma. However, recent data suggest the feasibility of omitting radiotherapy in patients of PMBCL with bulky disease treated with DA-EPOCH-R regimen. ${ }^{21}$

\section{Conclusion}

The challenging situation of a cancer diagnosis during pregnancy must be approached in a systematic and multidisciplinary manner. The necessary cautions must not become unreasonable. A pregnant woman with suspected malignancy who requires emergent radiographic imaging potentially faces risks arising from malignancy to her own health, as well to the health of the fetus. These risks usually outweigh the minor hazards posed by low-dose radiation exposure. Similarly, combination chemoimmunotherapy is feasible with the continuation of pregnancy in most cases, after the first trimester. In short, a multidisciplinary approach can lead to gratifying outcomes in many patients diagnosed with malignancy during pregnancy.

\section{Authors' Contribution}

$A A, A J, G P$, and RB managed the case. RS reported the pathology. AA and AJ wrote the paper.

\section{Declaration of Patient Consent}

The authors certify that they have obtained all appropriate patient consent forms. In the form the patient has given her consent for images and other clinical information to be reported in the journal. The patient understands that her name and initials will not be published and due efforts will be made to conceal her identity, but anonymity cannot be guaranteed.

\section{Funding}

Nil.

\section{Conflicts of Interest}

There are no conflicts of interest.

\section{Acknowledgments}

Rintu Sharma, Navneet Sharma, Pankaj Malhotra. Department of Internal Medicine, PGIMER, Chandigarh

\section{References}

1 Stensheim H, Møller B, van Dijk T, Fosså SD. Cause-specific survival for women diagnosed with cancer during pregnancy or lactation: a registry-based cohort study. J Clin Oncol 2009;27(1):45-51

2 Djakovic A, Ott G, Zollner U, Vordermark D, Dietl J. [Mediastinal large B-cell lymphoma with symptomatic superior vena cava syndrome in a patient with bichorial twin pregnancy in the 26th week of gestation: peri-and postpartal management - a case report]. Zentralbl Gynäkol 2005;127(4):248-251

3 Obeidat OS, Baniissa BA, Shkoukani ZW, Alhouri AN. Mediastinal lymphoma-induced superior vena cava syndrome and chylopericardium in a pregnant lady: a case report. Avicenna J Med 2019;10(2):89-92

4 Mangasarova JK, Kravchenko SK, Magomedova AU, Baryakh E, Vorobiev VI, Savchenko VG. Pregnancy and primary mediastinal B-cell lymphoma. Blood 2015;126(23):5069-5069

5 Ratnapalan S, Bona N, Chandra K, Koren G. Physicians' perceptions of teratogenic risk associated with radiography and CT during early pregnancy. AJR Am J Roentgenol 2004;182(5):1107-1109

6 Lowe S. Diagnostic imaging in pregnancy: making informed decisions. Obstet Med 2019;12(3):116-122

7 Barzilai M, Avivi I, Amit O. Hematological malignancies during pregnancy. Mol Clin Oncol 2019;10(1):3-9

8 De Santis M, Di Gianantonio E, Straface G, et al. Ionizing radiations in pregnancy and teratogenesis: a review of literature. Reprod Toxicol 2005;20(3):323-329

9 Streffer C, Shore R, Konermann G, et al. Biological effects after prenatal irradiation (embryo and fetus). A report of the International Commission on Radiological Protection. Ann ICRP 2003;33(1-2):5-206 [internet]

10 ACOG Committee on Obstetric Practice. ACOG Committee Opinion. Number 299, September 2004 (replaces No. 158, September 1995). Guidelines for diagnostic imaging during pregnancy. Obstet Gynecol 2004;104(3):647-651

11 Gjelsteen AC, Ching BH, Meyermann MW, et al. CT, MRI, PET, PET/CT, and ultrasound in the evaluation of obstetric 
and gynecologic patients. Surg Clin North Am 2008;88(2): 361-390, vii vii.

12 Tremblay E, Thérasse E, Thomassin-Naggara I, Trop I. Quality initiatives: guidelines for use of medical imaging during pregnancy and lactation. Radiographics 2012;32(3):897-911

13 Groen RS, Bae JY, Lim KJ. Fear of the unknown: ionizing radiation exposure during pregnancy. Am J Obstet Gynecol 2012;206(6):456-462

14 Committee on Obstetric Practice. Committee Opinion No. 723: Guidelines for diagnostic imaging during pregnancy and lactation. Obstet Gynecol 2017;130(4):e210-e216

15 Pereg D, Koren G, Lishner M. The treatment of Hodgkin's and non-Hodgkin's lymphoma in pregnancy. Haematologica 2007;92(9):1230-1237

16 Brenner B, Avivi I, Lishner M. Haematological cancers in pregnancy. Lancet 2012;379(9815):580-587
17 Evens AM, Advani R, Press OW, et al. Lymphoma occurring during pregnancy: antenatal therapy, complications, and maternal survival in a multicenter analysis. J Clin Oncol 2013;31(32):4132-4139

18 Gziri MM,DebièveF,DECatte L, etal.Chemotherapy during pregnancy: effect of anthracyclines on fetal and maternal cardiac function. Acta Obstet Gynecol Scand 2012;91(12):1465-1468

19 Giulino-Roth L. How I treat primary mediastinal B-cell lymphoma. Blood 2018;132(8):782-790

20 Malenda A, Kołkowska-Leśniak A, Puła B, et al. Outcomes of treatment with dose-adjusted EPOCH-R or R-CHOP in primary mediastinal large B-cell lymphoma. Eur J Haematol 2020;104(1):59-66

21 Chan EHL, Koh LP, Lee J, et al. Real world experience of R-CHOP with or without consolidative radiotherapy vs DA-EPOCH-R in the first-line treatment of primary mediastinal B-cell lymphoma. Cancer Med 2019;8(10):4626-4632 